\title{
Robotics in Surgery - Past, Present and Future
}

\section{Rahul G* and Abhishek C}

Department of General Surgery, PGIMER, Chandigarh, India

The first description of use of surgical robot was for taking neurosurgical biopsies with precision using PUMA 560 model in 1985 [1]. Subsequently another robotic system was designed so as to assist in performing transurethral resection called as PROBOT. A few years later, Integrated Surgical Systems developed a robotic system called ROBODOC in 1992 [2]. This robot was mainly used in orthopaedics where it was found to be useful in accurate implantation of prosthesis in knee and hip arthroplasty procedures. As these surgical robots were found to be very useful in assisting various procedures, need was felt to develop robot which could assist in holding laparoscopic camera. And soon a surgical robot came in to the market after FDA approval in 1994 by the name of AESOP [Automated Endoscopic System for Optimal Positioning] [3]. This was first of its kind robotic system which allowed the operating surgeon to control the position of the endoscope using either voice commands or a foot pedal. With further advancement in technology, first master slave robot called ZEUS robotic system was developed in 1998 which not only allowed the surgeon to control the endoscope but also provided platform to perform surgery from remote distance using manipulating robotic arms. Subsequently this robotic system was further refined and modified to develop da Vinci Surgical System which is the most widely used surgical robot in today's surgical practice.

This currently used robotic system has several advantages over open or laparoscopic surgery which includes 3D high definition magnified vision, tremor filtration, and better ergonomics with the use of Endowrist instruments which provide 540 degrees of freedom. It has been used by all the subspecialties of surgical field including cardiovascular surgeries, gastrointestinal surgeries. But these theoretical advantages have failed to translate in to better postoperative outcomes universally. It has been found to be most useful in urological and gynaecological cases with respect to better post operative outcomes. Various other robotic systems like the NeuroArm, Spine Assist and Renaissance have been developed for neurosurgery, spine surgery where da Vinci system has limitations.

In order to reduce postoperative pain, improve cosmesis, single incision laparoscopic surgery was developed as a step towards being more minimally invasive. But this technique suffers from some major drawbacks like poor ergonomics, lack of triangulation, crowding of instruments preventing its wide spread use. In order to overcome these deficiencies, Intuitive Surgical Inc. designed special set of instruments and multichannel access port. This port has two curved cannulae for placing robotic instruments, one channel for endoscope, one bedside assistant surgeon port and an insufflation valve.

But the major drawbacks of the existing robotic system like da Vinci system which is preventing their widespread use are the lack of haptic feedback, bulky set up, additional docking time and high cost. Inorder to provide haptic feedback many new accessories like haptic hand controllers, haptic pedal have been tested in experimental studies and have shown promising results [4,5]. With future advances in technology, we are likely to see surgical robots which are less bulky and more flexible. Technology is advancing at rapid pace and there has been development of specialized flexible endoscopic robot which will help in performing complex gastrointestinal surgeries using NOTES technique [6]. New generation surgical robots like cognitive surgical robots, modular miniature robots are likely to take robotic surgery to altogether new standards $[7,8]$. The cost of robotic surgery is likely to reduce in future with reduction in cost of research, manufacturing and marketing.

At present it would be desirable to say that the technology of surgical robots is evolving and advancing at rapid pace and is likely to make robotic assisted surgery more efficient with better postoperative outcomes and cosmesis in future.

\section{References}

1. Kwoh YS, Hou J, Jonckheere EA, Hayati S (1988) A robot with improved absolute positioning accuracy for CT guided stereotactic brain surgery. IEEE Trans Biomed Eng 35: 153-60.

2. Satava RM (2002) Surgical robotics: The early chronicles: A personal historical perspective. Surg Laparosc Endosc Percutan Tech 12: 6-16.

3. Unger SW, Unger HM, Bass RT (1994) AESOP robotic arm. Surg Endosc 8 11-31.

4. Díaz I, Gil JJ, Louredo M (2014) A haptic pedal for surgery assistance. Comput Methods Programs Biomed 116: 97-104.

5. Zareinia K, Maddahi Y, Ng C, Sepehri N, Sutherland GR (2015) Performance evaluation of haptic hand-controllers in a robot-assisted surgical system. Int $J$ Med Robot.

6. Lomanto D, Wijerathne S, Ho LK, Phee LS (2015) Flexible endoscopic robot Minim Invasive Their Allied Technol 24: 37-44

7. Zygomalas A, Giokas K, Koutsouris D (2014) In silico investigation of a surgical interface for remote control of modular miniature robots in minimally invasive surgery. Minim Invasive Surg, Epub.

8. Kassahun Y, Yu B, Tibebu AT, Stoyanov D, Giannarou S, et al. (2015) Surgical robotics beyond enhanced dexterity instrumentation: A survey of machine learning techniques and their role in intelligent and autonomous surgical actions. Int J Comput Assist Radiol Surg.
*Corresponding author: Rahul G, Department of General Surgery, PGIMER,
Chandigarh, India, Tel: +917087009649; E-mail: rahul.g.85@gmail.com

Received November 17, 2015; Accepted November 20, 2015; Published November 22, 2015

Citation: Rahul G, Abhishek C (2015) Robotics in Surgery - Past, Present and Future. Adv Robot Autom S2:e001. doi:10.4172/2168-9695.S2-e101

Copyright: ( 2015 Rahul G, et al. This is an open-access article distributed unde the terms of the Creative Commons Attribution License, which permits unrestricted use, distribution, and reproduction in any medium, provided the original author and source are credited. 